\title{
MUCOUS COLITIS.
}

By J. BROWNING ALEXANDER, M.D., M.R.C.P.

(Senior Physician, Prince of Wales's General Hospital. Physician, London Chest Hospital. Physician, St. Mark's Hospital.)

In this article my intention is to deal merely with that form of colitis known as mucous colitis, or muco-membranous entero-colitis. Before discussing the ætiology or pathology of this disease, I shall endeavour to describe what I consider to be a typical picture of the sufferer from this condition.

The patient is usually a woman somewhere between the ages of 25 and 40 . She wears a worried and distressed appearance, is usually pale and haggard, with a muddy complexion. She is invariably neurotic and has become a burden to herself and others around her. She complains of constant abdominal discomfort with periods of intense pain of a spasmodic or colicky nature. Dyspepsia is a constant feature of her varied symptoms, the dyspepsia is usually of the flatulent type, and the history can usually be obtained of obstinate constipation, alternating occasionally with attacks of diarrhœa. On questioning the sufferer one will learn that from time to time mucus is passed from the bowel, sometimes in small amounts consisting only of shreds, but at other times in large quantities. It is usually, one is told, after severe paroxysms of pain that the larger quantity is present. One may obtain also the history of evacuation of blood. This in large amount is infrequent, but the history of smaller quantities, amounting to not more than streaks, is usually forthcoming. This condition may have been going on for a considerable length of time before the patient seeks advice regarding the intestinal trouble. The onset is indefinite in many cases and one is often at a loss to ascertain the mode of commencement of the trouble. It may be significant, however, that not infrequently the patient will vouchsafe the information that the passage of mucus has been present for a considerably longer time than the actual symptoms of discomfort or pain have existed. Occasionally the mucus may be passed in the form of cylindrical masses, which may be regarded as casts of the bowel.

When presented with such a picture as that of the foregoing, one would have little hesitation in making the diagnosis of mucous colitis; but having thus labelled it, one has not necessarily arrived at any real conception of the true nature of the morbid processes concerned.

What is mucous colitis? Can it be regarded as a disease by itself, or merely a symptom? Is it a pure neurosis of the secretory type and can it be put into line with such other conditions as asthma, croup, etc. ?

In support of the theory that it be a manifestation of neurosis is the fact that all patients suffering from the disease are, one might say, invariably neurotic. Furthermore, additional weight is given to this contention by the failure usually experienced at autopsy (the patient having died of some intercurrent malady) to find in the colon any pathological changes.

The neurotic conception cannot fail to appeal to one as unsatisfactory and unscientific. As far as I know, no convincing proof has been put forward to explain why functional derangement of the colon, giving rise to the symptoms already described, should be caused by a primary neurosis. Sufferers from any chronic or longstanding abdominal condition not unnaturally show at some period or other a tendency to neurosis, but rarely has one seen a cure effected or even any amelioration of the symptoms by attention to the nervous side of the picture only. 
Furthermore, pathological changes have been not infrequently noted by surgeons during the course of abdominal operations; they have reported extensive chronic catarrhal inflammation of the mucous membrane of the colon. One is probably on safer ground in regarding mucous colitis as a chronic catarrhal inflammation of the colon, secondary to some abnormal intra-abdominal state.

As examples of the intra-abdominal conditions which we associate with the symptom complex of mucous colitis, the following may be mentioned as those commonly met with :-

(I) Peritoneal adhesions resulting from old abdominal operations. Not infrequently in patients suffering from mucous colitis one observes more than one abdominal scar and a history is obtained that an operation for some abdominal condition had been performed many years before, and that subsequent laparotomies had been undertaken to free adhesions which were deemed to have arisen from the first operation. In many instances the freeing of such adhesions had resulted in further ones being produced. Such adhesions must be regarded as of a conservative type rather than of an inflammatory nature.

(2) In association with intra-abdominal peritoneal adhesions and particularly after these "conservative" adhesions have been cut, a state of visceroptosis is likely to develop, giving rise to kinking of different parts of the colon and consequent local catarrhal inflammation of its lining membrane. The ill nourished state of the colitis patient tends to exaggerate still more the ptosis, and a vicious circle is established, by which the greater the degree of visceroptosis, the greater the severity of the colitis and vice versa.

(3) A diseased appendix is not uncommonly found to be associated with? mucous colitis and instances are recorded in which the removal of the diseased appendix has brought about a cure of the colitis. It is doubtful whether inflammation spreads directly from the appendix to the cæcum and ascending colon, or whether the appendix merely furnishes a focus of infection and brings about an inflammation of the colon in a similar manner to a septic focus elsewhere. For example, a focus of infection in teeth or tonsils is often responsible for inflammatory mischief in other parts of the body.

(4) Disease of the uterus or its appendages, or even displacement of the organ has in some cases been known to be associated with mucous colitis, and I have personal experience of one case of mucous colitis which was apparently cured after a retroverted uterus had reeceived attention.

(5) Malignant disease of some part of the colon-particularly of the sigmoid -has on occasions produced all the symptoms of a well-marked mucous colitis before arousing suspicion with regard to the true nature of the disease. It is of the utmost importance, therefore, to keep in mind this possibility, and in all cases presenting the symptoms of colitis to exclude or otherwise the presence of carcinoma.

It is possible that all these conditions mentioned above bring about a state of mucous colitis through the agency of constipation, and some people regard mucous colitis as being merely the combination of a state of chronic constipation, and a secondary neurosis; but there appears also to be some evidence that infection may actually spread from the diseased or altered part and be responsible for the colon inflammation. 
Having already described what I consider a typical clinical picture of the sufferer from mucous colitis, nothing more need be added regarding the symptoms of this condition, and with regard to the physical signs-these are often very indefinite. As a rule the patient is hypersensitive and complains of tenderness in almost every region of the abdomen. The want of localization of pain or tenderness in itself is a significant sign of the disease. The abdomen is usually flabby and the abdominal wall thin. The coils of the intestines are often visible, and occasionally one can detect on palpation a thickened colonic wall. When visceroptosis is present (and this is frequent) one will be able to feel the dropped kidney and appreciate a ptosis of the abdominal organs generally. During an attack of colic the abdomen occasionally becomes distended and tender and occasionally a tumour mass may be felt in the large intestine, probably due to the rolling up of mucus inside the lumen of the bowel. A few years ago I saw a case of this kind in which the mucus tumour had been mistaken for a malignant growth.

The evacuations are not necessarily frequent except during attacks of colic, when diarrhœa may be present. In the intervals between attacks constipation is the rule, and with the constipated motion shreds of mucus or small casts, and occasional streaks of blood are seen.

\section{Diagnosis.}

The symptoms which I have described are usually in themselves sufficient to make the diagnosis. The underlying cause of the condition, however, is not always so simple of elucidation. It is important to bear in mind the various predisposing causes and make a careful search for their presence.

Such a condition as dysentery is not likely to be confounded with mucous colitis. In the former the tenesmus and non-fæcal characteristics of the motions should usually suffice to make the diagnosis, and where any suspicion may still linger in one's mind of its presence, a complete bacteriological examination of the stools will render the diagnosis clear.

Ulcerative colitis may occasionally be mistaken for mucous colitis, but in that form the large quantities of blood passed will suggest the true diagnosis and a sigmoidoscopic examination will in most cases establish the true morbid state.

I have already referred to malignant disease in association with mucous colitis, and I would again emphasize the importance of bearing this possibility in mind and regarding it as one's duty to make the fullest investigations possible by every means at our disposal to avoid missing the graver disease.

A more unlikely mistake in the differential diagnosis of colitis is that of tuberculous peritonitis. This condition is but rarely met with in adults. It is more common in children, and when it does occur in adults some other evidence of tuberculosis is usually present.

\section{Prognosis.}

This would depend to a great extent upon an accurate diagnosis having been made. Should one of the underlying causes which I have outlined be found and dealt with, the outlook as regards recovery is usually good. When no such underlying cause can be detected and the case is deemed to be one of well established neurosis associated with general emaciation and visceroptosis, the patient is often very resistant to any form of treatment and such cases often persist for years. 


\section{Treatment.}

It is useless to attempt any form of treatment without trying first to discover one of the primary causes already named which are known to be responsible for the disease. Thus a diseased appendix should be removed, a displaced uterus readjusted, or an abdominal support applied to correct marked downward displacement of organs. Surgical measures calculated to relieve the visceroptosis, such as nephropexy, etc. are not to be recommended and peritoneal adhesions, unless they be causing obstruction, are better left alone.

There are many cases, however, in which no primary cause exists or where at least none is found. In these cases some special line of treatment must be adopted which will improve the general condition of the patient and relieve the symptoms of the catarrhal inflammatory process of the colon.

The old time favoured plan of feeding the patient on slops and milk is to be deprecated. Patients suffering from this condition are usually emaciated and poorly nourished, not merely as the result of the disease, but in many instances due to a voluntary cutting down of the diet, believing that certain articles of food are responsible for the symptoms. In such patients a vicious circle is establishedviz. the greater the starvation the more pronounced become the symptoms, and as the symptoms increase in severity, the greater is the starvation. In such patients a more generous diet is required, but it is often, if not nearly always, impossible to enhance the diet to any great extent at once, a gradual supplementing of it being usually the wiser procedure. Fats, which the patient has usually avoided, are actually in the majority of cases well tolerated and can be given in the form of cream, butter, or bacon fat. Farinaceous soups are also usual and are more often employed on the Continent than in England.

Eggs in the form of omelettes or buttered can be given, and gradually one may augment the dietary still further by the addition of such articles of food as fish, chicken and meat. The diet should be abundant, and for this reason cellulous and other coarse materials are of advantage, such as fruit, vegetables and brown bread - but it must be remembered that fruit and vegetables which are liable to leave a residue or are notably indigestible must be avoided-at any rate in the early stages of treatment. One's aim should be to produce bulky motions, at the same time avoiding as far as possible constipation. The latter can be obviated to some extent by as liberal an amount of fat as possible, which, as I have already stated, is well tolerated by colitis patients. It should be borne in mind that only a certain amount of fat is capable of being absorbed by the colon, therefore a certain proportion of it must be excreted and will act as a lubricant and further will tend to protect the intestinal mucosa from irritation.

Drugs. It is of the utmost importance to maintain a regular action of the bowels, but drastic purgatives should be avoided. It is often difficult to convince the sufferer that better results will be obtained from simple aperients. Liquid paraffin, which is not a purgative but a lubricant, given regularly two or three times daily is often sufficient to ensure regular bowel action, but in cases where something more is required, castor oil is of advantage. Personally I have found more use from such preparations as petrolagar or semprolin. During the acute attacks of severe colic drugs must be employed and it is often necessary to administer a hypodermic injection of morphia-but I have found that in most cases belladonna given by the mouth or an injection of atropine is equally efficacious and undoubtedly these are safer drugs to employ. On several occasions I have 
found elixir papaine in I drachm doses of use. Since the patient, as already stated, is usually emaciated and has been on a semi-starvation diet, iron is deficient and can be with advantage prescribed in conjunction with nux vomica. Intestinal antiseptics have from time to time been advocated, but personally I have found them of little use, relying more on the avoidance of constipation as a means to keep the intestinal canal clear.

Rectal Injections. Colonic lavage is a popular form of treatment for colitis and probably used more extensively abroad than at home. Normal saline may be used or in some cases an astringent such as nitrate of silver. My own experience has led me to avoid giving astringents if possible; as I feel sure that in many instances they are apt to create further bowel irritation. In my own experience I have relied more on the injection of warm olive oil, giving $\frac{1}{2}-I$ pint into the rectum at night, the injection being made slowly and an endeavour made by the patient to retain it for as many hours as possible. The majority of patients can retain this during the whole night. This should be followed by a warm water enema in the morning. In my opinion it is unwise to continue this treatment over too long a period and my usual practice is to recommend the injections to be used for four weeks, if necessary repeating a similar course at a later date.

Massage. As a rule a considerable amount of benefit can be derived from a certain amount of massage, as the patients invariably have very flabby abdominal muscles, In my opinion, however, the mistake usually made is to overdo this form of treatment. I have more than once observed extreme exhaustion in a patient after having been subjected to half an hour's vigorous massage in the early stages of treatment. I think it a wise plan to instruct the masseur or masseuse to give not more than ten minutes at the commencement and to increase by a few minutes each time. It is also important to limit the duration of the whole treatment. Two or three weeks of daily massage is usually sufficient and it can be repeated after a few weeks' interval for a similar period. In some cases at a later date a little electrical current applied to the abdomen can be combined with the massage, and later (particularly if visceroptosis exists) a suitable abdominal support should be prescribed. A properly designed abdominal support should exert pressure in an upward and backward direction. An ill-fitting and improperly designed belt is likely to do more harm than good.

Vaccines. Various attempts have been made at different times to treat colitis by means of vaccines. The organisms usually concerned are varieties of the colon bacillus and the streptococcus. Cases are on record in which it is claimed that benefit has been derived from such therapy. Personally I have never seen good results from this form of treatment and have entirely given up its use. 